\title{
Deterministic cavity-QED source of polarization-entangled photon pairs
}

\author{
R. García-Maraver, K. Eckert, R. Corbalán, and J. Mompart \\ Departament de Física, Universitat Autònoma de Barcelona, E-08193 Bellaterra, Spain
}

(Received 27 January 2006; published 28 September 2006)

\begin{abstract}
We present two cavity quantum electrodynamics proposals that, sharing the same basic elements, allow for the deterministic generation of entangled photon pairs by means of a three-level atom successively coupled to two single longitudinal mode high- $Q$ optical resonators presenting polarization degeneracy. In the faster proposal, the three-level atom yields a polarization-entangled photon pair via two truncated Rabi oscillations, whereas in the adiabatic proposal, a counterintuitive stimulated Raman adiabatic passage process is considered. Although slower than the former process, this second method is very efficient and robust under fluctuations of the experimental parameters and, particularly interesting, almost completely insensitive to atomic decay.
\end{abstract}

DOI: 10.1103/PhysRevA.74.031801

The main issue in cryptography is the secure distribution of the encoding key between two partners. With this aim, quantum cryptography renders two classes of protocols [1-3] based, respectively, on superposition and quantum measurement, or entanglement and quantum measurement. Entanglement-based protocols, first considered by Ekert [3], present some potential advantages: (i) under passive state preparation, frustration of multiphoton splitting attacks occurs since each photon pair is uncorrelated from the rest [4]; (ii) in the presence of dark counting, entangled states allow for the detection and removal of empty photon pulses by means of coincidence photodetection; (iii) for some entangled states lying in decoherence-free subspaces, no information flows to nonrelevant degrees of freedom [5]; and, for quantum networks, (iv) there is null information leakage to the provider of the key. In spite of (i), it is important to have single-photon pairs since multiphoton pairs decrease the quantum correlations between the measurement results and, accordingly, enhance the quantum bit error rate [6].

Quantum cryptography with entangled states has been achieved by means of parametric downconverted photons generated in nonlinear crystals [7-9]. However, in all these cases the photon number statistics (and their time distributions) follows, essentially, a Poissonian distribution. Thus, in order to reduce the number of multiphoton pairs, the average photon number has to be much less than 1 , which, in turn, strongly reduces the key exchange rate. Accordingly, one of the practical issues in entanglement-based quantum cryptography presently attracting considerable attention is the development of light sources that emit deterministically single entangled photon pairs at a constant rate [10-12]. In addition, it is worthwhile to notice that single pairs of entangled photons and more involved nonclassical photon states [13] have a fundamental significance for testing quantum mechanics against local hidden variable theories and for practical applications in teleportation [14] and dense coding [15].

Focusing on the optical regime, we discuss here a cavityquantum-electrodynamics (CQED) implementation [16-23] that, making use of a V-type three-level atom coupled successively to two high- $Q$ cavities presenting polarization degeneracy, allows for the deterministic generation of polarization-entangled photon pairs. Two different proposals are investigated for the entangled photon pair generation based, respectively, on two truncated Rabi oscillations (ROs) and on a stimulated Raman adiabatic passage (STIRAP) pro-
PACS number(s): 42.50.Pq, 03.67.Dd, 03.67.Mn, 32.80.-t

cess [24]. The feasibility of both proposals and some practical considerations will be discussed for realistic parameter values of state-of-the-art experiments in optical CQED [18-23].

The system under investigation is sketched in Fig. 1 and is composed of a single $\mathrm{V}$-type three-level atom with two electric dipole transitions of frequencies $\omega_{a c}$ and $\omega_{b c}$, and two high- $Q$ cavities both displaying polarization degeneracy and having identical longitudinal mode frequency $\omega_{c} . \Delta_{+}$ $=\omega_{c}-\omega_{a c}$ and $\Delta_{-}=\omega_{c}-\omega_{b c}$ are the detunings. The transition $\left|a, n_{i+}\right\rangle \leftrightarrow\left|c, n_{i+}+1\right\rangle\left(\left|b, n_{i-}\right\rangle \leftrightarrow\left|c, n_{i-}+1\right\rangle\right)$ will be governed by the coupling $g_{i+} \sqrt{n_{i+}+1}\left(g_{i-} \sqrt{n_{i-}+1}\right)$ with $i=1,2$ denoting the cavity, $g_{i \pm}$ the vacuum Rabi frequency of the corresponding circular polarization, and $n_{i \pm}$ the number of $\sigma_{ \pm}$circularly polarized photons. We will consider here the completely symmetric case given by $\omega_{a c}=\omega_{b c}, \Delta_{+}=\Delta_{-}(\equiv \Delta)$, and $g_{i+}(t)=g_{i-}(t)\left[\equiv g_{i}(t)\right]$. This symmetry could be easily obtained by considering a $J=0 \leftrightarrow J=1$ atomic transition with the quantization axis along the optical axis of the cavities. Eventually, we will relax some of the previous symmetry requirements in analyzing the influence of experimental imperfections.
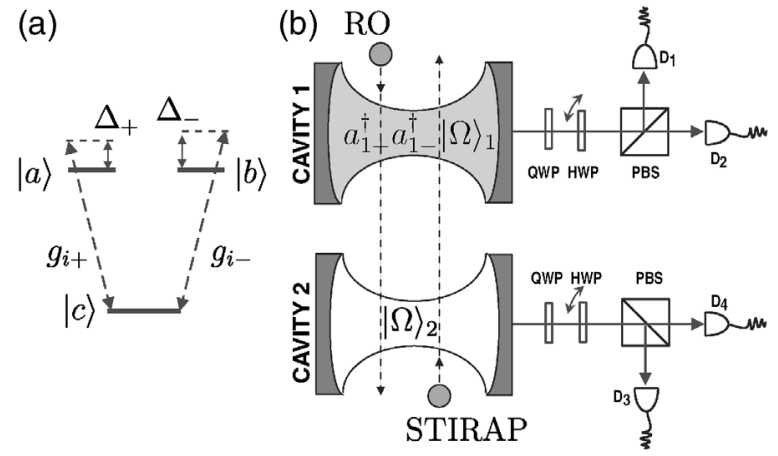

FIG. 1. (a) V-type three-level atomic configuration under investigation. (b) Proposed setup for the deterministic generation of polarization entangled photon pairs. $|\Omega\rangle_{i}$ is the two-mode vacuum state of cavity $i$. The two proposed configurations based, respectively, on two truncated ROs and STIRAP are simultaneously shown. Also shown are the basic optical elements needed for the Bell analysis of the entangled states (QWP, quarter-wave plate; HWP, rotating half-wave plate; PBS, polarization beam splitter; $D_{i}$, single-photon detector). 
(a)

(b)

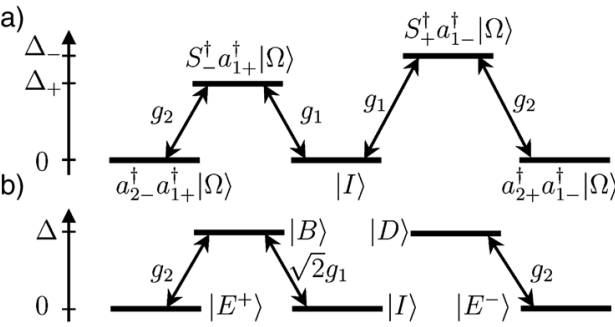

FIG. 2. (a) Manifold of states coupled to $|I\rangle=a_{1_{+}}^{\dagger} a_{1-}^{\dagger}|\Omega\rangle$ and the corresponding relative energies and coupling strengths (in the interaction picture). (b) The same manifold in terms of the basis states given in Eqs. (4)-(8) under the two-photon resonance condition.

In the rotating-wave approximation, the coherent dynamics of the full system is described by the Hamiltonian

$$
\begin{gathered}
H=H_{0}+H_{I}, \\
H_{0}=\sum_{i=1,2} \omega_{c}\left(a_{i+}^{\dagger} a_{i+}+a_{i-}^{\dagger} a_{i-}\right)+\sum_{j=a, b} \omega_{j c}|j\rangle\langle j|, \\
H_{I}=\sum_{i=1,2} g_{i}\left(a_{i+}^{\dagger} S_{+}+a_{i+} S_{+}^{\dagger}+a_{i-}^{\dagger} S_{-}+a_{i-} S_{-}^{\dagger}\right)
\end{gathered}
$$

$(\hbar=1)$, where $a_{i \pm}^{\dagger}\left(a_{i \pm}\right)$ is the photon creation (annihilation) operator in the corresponding cavity mode, $S_{+}=|c\rangle\langle a|$, and $S_{-}=|c\rangle\langle b|$.

The couplings given in Eq. (3) allow us to group the states of the full system composed of the atom plus the cavity modes into decoupled manifolds. We assume the ability to prepare the intracavity fields in a Fock state [17] and take $|\psi(t=0)\rangle=a_{1+}^{\dagger} a_{1-}^{\dagger}|\Omega\rangle(\equiv|I\rangle)$ as the initial state of the system with $|\Omega\rangle \equiv|c\rangle \otimes|\Omega\rangle_{1} \otimes|\Omega\rangle_{2}$, where $|\Omega\rangle_{i}$ is the two-mode vacuum state of cavity $i$. In this case, the coherent evolution of the system is constrained to remain in the space spanned by the five states of the manifold shown in Fig. 2(a). Let us consider an alternative basis of this manifold given by

$$
\begin{gathered}
|I\rangle \equiv a_{1_{+}}^{\dagger} a_{1-}^{\dagger}|\Omega\rangle, \\
\sqrt{2}|B\rangle \equiv\left(S_{+}^{\dagger} a_{1_{-}}^{\dagger}+S_{-}^{\dagger} a_{1+}^{\dagger}\right)|\Omega\rangle, \\
\sqrt{2}|D\rangle \equiv\left(S_{+}^{\dagger} a_{1-}^{\dagger}-S_{-}^{\dagger} a_{1_{+}}^{\dagger}\right)|\Omega\rangle, \\
\sqrt{2}\left|E^{ \pm}\right\rangle \equiv\left(a_{2_{+}}^{\dagger} a_{1_{-}}^{\dagger} \pm a_{2}^{\dagger} a_{1_{+}}^{\dagger}\right)|\Omega\rangle .
\end{gathered}
$$

$|B\rangle$ and $|D\rangle$ are the so-called bright and dark states [26] resulting from the combination of the excited atomic states and the modes of cavity $1 .\left|E^{ \pm}\right\rangle$correspond to two Bell states for the photons while the atomic state factorizes. In the interaction picture, it is straightforward to check that, under the two-photon resonance condition, $\Delta_{+}=\Delta_{-}(\equiv \Delta)$, the latter basis states satisfy $\langle D|H| I\rangle=\left\langle D|H| E^{+}\right\rangle=\left\langle B|H| E^{-}\right\rangle=0,\langle B|H| I\rangle$ $=\sqrt{2} g_{1} e^{-i \Delta t},\left\langle B|H| E^{+}\right\rangle=\left\langle D|H| E^{-}\right\rangle=g_{2} e^{-i \Delta t}$. The resulting coupling chain is schematically illustrated in Fig. 2(b) and suggests the two proposals of this paper.

Proposal 1: The two ROs scheme. Interaction starts in cavity 1 with two different pathways for the atomic excita- tion, from $|I\rangle$ to $S_{+}^{\dagger} a_{1-}^{\dagger}|\Omega\rangle$ and to $S_{-}^{\dagger} a_{1+}^{\dagger}|\Omega\rangle$ [see Fig. 2(a)]. However, under the two-photon resonance condition, one indeed deals with an effective two-level system where ROs occur between states $|I\rangle$ and $|B\rangle$ [Fig. 2(b)]. In the interaction picture, the solution of the Schrödinger equation for $g_{2}=0$ yields

$$
\begin{aligned}
|\psi(t)\rangle= & e^{-i \Delta t / 2}\left[\left(-i 2 \sqrt{2} g_{1} / \Omega_{1}\right) \sin \left(\Omega_{1} t / 2\right)\right]|B\rangle \\
& +e^{i \Delta t / 2}\left[\cos \left(\Omega_{1} t / 2\right)-i\left(\Delta / \Omega_{1}\right) \sin \left(\Omega_{1} t / 2\right)\right]|I\rangle,
\end{aligned}
$$

where $\Omega_{1}=\sqrt{8 g_{1}^{2}+\Delta^{2}}$ is the so-called generalized quantum Rabi frequency. Hence, under the single-photon resonance condition, $\Delta=0$, there are complete population oscillations between these two states.

With this dynamics in mind, the steps to generate deterministically a polarization-entangled photon pair are as follows. (i) Preparation of the system into the initial state $|I\rangle$. A regular temporal distribution of atoms entering the setup in state $|c\rangle$ could be realized by means of selective laser excitation. (ii) The three-level atom interacts resonantly with the two circular polarizations modes of cavity 1 for an interaction time, $t_{1}$, yielding half-of-a-resonant RO with the bright state, i.e., $\int_{0}^{t_{1}} \Omega_{1}(t) d t=\pi$, with $\Omega_{1}(t)=2 \sqrt{2} g_{1}(t)$ [25]. The state of the system after this step is $\left|\psi\left(t_{1}\right)\right\rangle=-i|B\rangle$. (iii) The threelevel atom couples to cavity 2 for a time $t_{2}$ such that $\int_{t_{1}}^{t_{1}+t_{2}} \Omega_{2}(t) d t=\pi$, with $\Omega_{2}(t)=2 g_{2}(t)$. If so, the vaccum modes of cavity 2 stimulate the emission of a single photon through the two paths $S_{+}^{\dagger} a_{1-}^{\dagger}|\Omega\rangle \rightarrow a_{2_{+}}^{\dagger} a_{1-}^{\dagger}|\Omega\rangle$ and $S_{-}^{\dagger} a_{1_{+}}^{\dagger}|\Omega\rangle$ $\rightarrow a_{2-}^{\dagger} a_{1+}^{\dagger}|\Omega\rangle$. The state of the system after this last step will be $\left|\psi\left(t_{1}+t_{2}\right)\right\rangle=-\left|E^{+}\right\rangle$. Hence, the state of the three-level atom factorizes and, in the end, each cavity contains exactly one photon. These two photons are entangled in their polarization degree of freedom.

Proposal 2: The STIRAP scheme. By diagonalizing Hamiltonians (1)-(3) in the interaction picture and assuming the two-photon resonance condition, it results that one of the energy eigenstates of the system is

$$
|\Lambda(\theta)\rangle=\cos \theta|I\rangle-\sin \theta\left|E^{+}\right\rangle,
$$

where the mixing angle $\theta$ is defined as $\tan \theta(t)$ $\equiv \sqrt{2} g_{1}(t) / g_{2}(t)$. Following Eq. (8), it is possible to transfer the system from $|I\rangle$ to $\left|E^{+}\right\rangle$by adiabatically varying the mixing angle from $0^{\circ}$ to $90^{\circ}$ realizing a counterintuitive STIRAP process [24]. In this case, the steps to generate the polarization-entangled photon pair are (i) preparation of the system into the initial state $|I\rangle$; (ii) the three-level atom couples first to the empty modes of cavity 2 ; and, before this interaction ends, (iii) the three-level atom starts to slowly interact with the modes of cavity 1 . Note that this last step means that the transverse spatial modes of the two cavities should appropriately overlap to assure the adiabaticity of the process. Although not as fast as the two truncated ROs proposal, the STIRAP process has two advantages: (i) it is very robust under fluctuations of the experimental parameters, e.g., interaction strengths and times, due to the fact that the system adiabatically follows an energy eigenstate; and (ii) it is almost not sensitive to atomic decay since, first, there is no 
need for single-photon resonance, and, second, $|\Lambda(\theta)\rangle$ never involves the intermediate state $|B\rangle$.

To further characterize this source of entangled photons, we consider the cavity decay of the photons through the mirrors and their eventual detection. Accordingly, we will investigate next the evolution of the system in the presence of two kinds of dissipative processes: first, spontaneous atomic decay from the two optical transitions at the common rate $\Gamma$; second, cavity decay of the photons through the mirrors and the irreversible process of their detection. We will assume a perfect quantum efficiency for the detectors $(\eta$ $=1$ ), and take the same mirror transmission coefficient, $\kappa$, for all four cavity modes. To account for dissipation, we have used the Monte Carlo wave function (MCWF) formalism [27] and averaged over many realizations of quantum trajectories. Accordingly, Hamiltonian (1)-(3) has been replaced by the following non-Hermitian Hamiltonian:

$$
H^{\prime}=H-i \frac{\Gamma}{2} \sum_{j=+,-} S_{j}^{\dagger} S_{j}-i \frac{\kappa}{2} \sum_{i=1,2}\left(a_{i+}^{\dagger} a_{i+}+a_{i-}^{\dagger} a_{i-}\right) .
$$

The evolution of the system in the presence of dissipative processes, obtained by averaging over many MCWF simulations, is shown in Figs. 3(a)-3(c). Figures 3(a) and 3(b) correspond to the two half-of-a-resonant ROs proposal and to the STIRAP case, respectively, with spontaneous decay being the dominant dissipative process. (c) accounts for the STIRAP scheme in the case of larger cavity decay of photons through the mirrors, with parameters corresponding to the best combination of atomic and cavity decay rates of stateof-the-art experiments in the optical domain [22,23]. For both schemes, the probabilities for different physical processes to happen are shown in Fig. 3(d) for the parameters from (c). Column (i) gives the success probability $P$ of producing $\left|E^{+}\right\rangle$after sending one atom through the setup. For parameters as in Figs. 3(a) and 3(b), $P=0.74$ and 0.83 , respectively, are obtained. In the STIRAP case, state $|B\rangle$ remains almost unpopulated for the whole process in spite of $\Delta_{+}=\Delta_{-}=0$, which makes it quite insensitive to atomic decay. For the parameters from Fig. 3(c), $P=0.41$ (RO) and $P=0.24$ (STIRAP). Here $P$ for the STIRAP scheme is lower since the process has to be adiabatic, i.e., significantly slower than the RO process, and it is thus more sensitive to cavity decay.

For both schemes, the source can be improved by postselection, i.e., by only keeping those events for which one photon from each cavity is registered in the detectors. We thus reduce to the space of two qubits defined by the polarizations of the photons and can accordingly define the fidelity as $F=\left\langle E^{+}|\rho| E^{+}\right\rangle$. In addition, we use the $S$ parameter of the CHSH inequality [28] to characterize the entanglement capability $(S=\sqrt{2}$ for any separable state and $S=2 \sqrt{2}$ for maximally entangled states). The results are summarized in Fig. 4 [29]. As visible from Fig. 4(b), for the STIRAP process the success probability is almost not affected by the atomic decay rate $\Gamma$. To further illustrate this enhanced robustness of the STIRAP compared to the RO scheme, Fig. 5 shows the success probability as a function of the atomic and cavity decay rates $(\mathrm{a}, \mathrm{b})$, and of the deviation from the single-
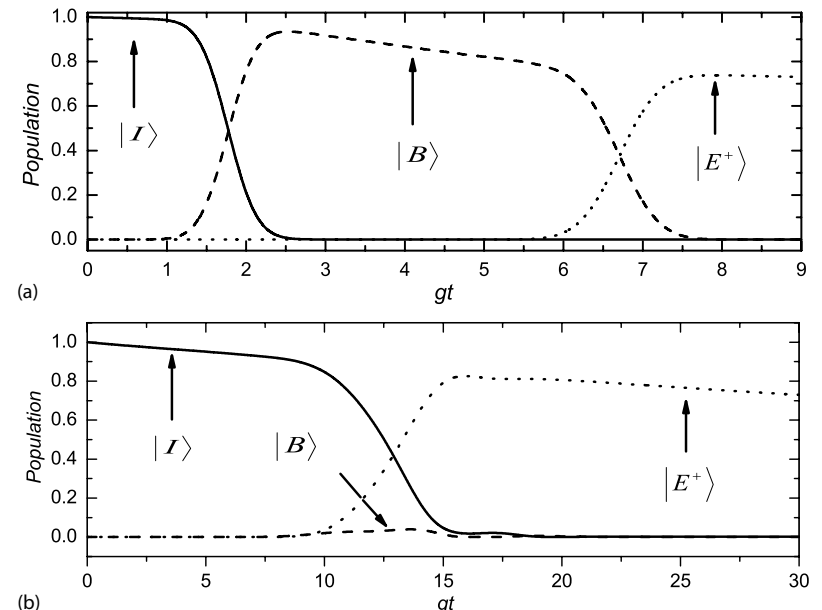

(b)
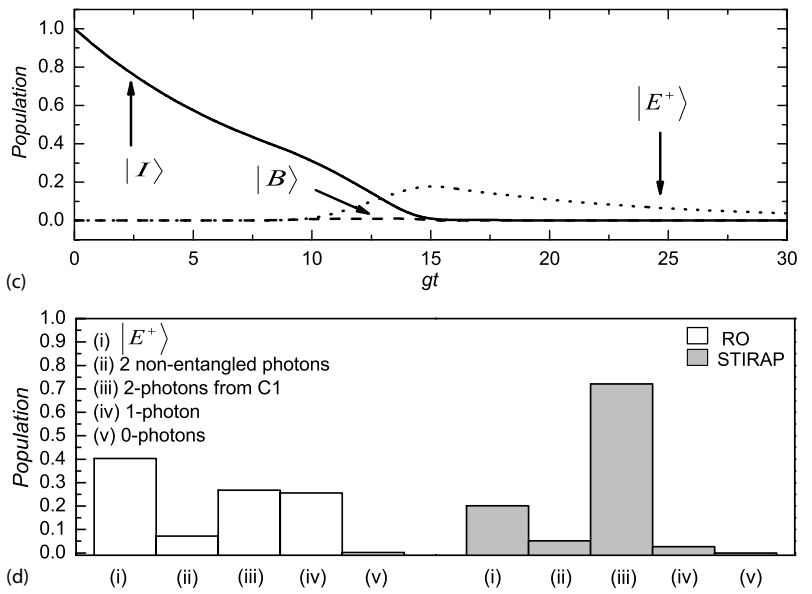

FIG. 3. Evolution of the system toward the entangled state $\left|E^{+}\right\rangle$ through two truncated ROs (a) and via STIRAP (b,c). $\Gamma=0.05 g$ and $\kappa=0.005 g$ for (a) and (b); $\Gamma=0.08 g$ and $\kappa=0.053 g$ for (c). In all cases, $\Delta_{+}=\Delta_{-}=0 . g$ is the vacuum Rabi frequency at the cavity center that we assume to be the same for both cavities. Realistic parameter values have been chosen for the Gaussian transverse profiles of the cavity modes. For STIRAP, appropriate overlapping between the transverse modes of the two cavities has been considered. (d) Probabilities for the different processes involving photon emission through the cavity mirrors and their eventual photodetection, for the RO as well as for the STIRAP scheme. Parameters are as in (c). Note that in (a)-(c), the height of column (i), i.e., the success probability $P$, corresponds to the maximum of the dotted line.

and two-photon resonance conditions (c,d). Figures 4(c) and 4(d) account, e.g., for the presence of a stray magnetic field such that $\left(\Delta_{+}-\Delta_{-}\right) / 2 \neq 0$, or an electric field yielding $\left(\Delta_{+}\right.$ $\left.+\Delta_{-}\right) / 2 \neq 0$. For example, for a $J=0$ to 1 transition, a magnetic field of $1 \mathrm{G}$ would reduce the fidelity of the CQED source by around $30 \%$ for the ROs proposal, while in the STIRAP proposal it would be reduced by only $3 \%$.

In conclusion, we have presented two schemes for the deterministic generation of polarization-entangled photon pairs based on a half-of-a-resonant RO in each cavity and on a STIRAP sequence, respectively. Both proposals depend differently on experimental imperfections: the RO scheme is likewise affected by decay of the excited atomic states and by cavity decay, while the STIRAP scheme is almost insensitive to atomic decay but affected strongly by cavity decay 


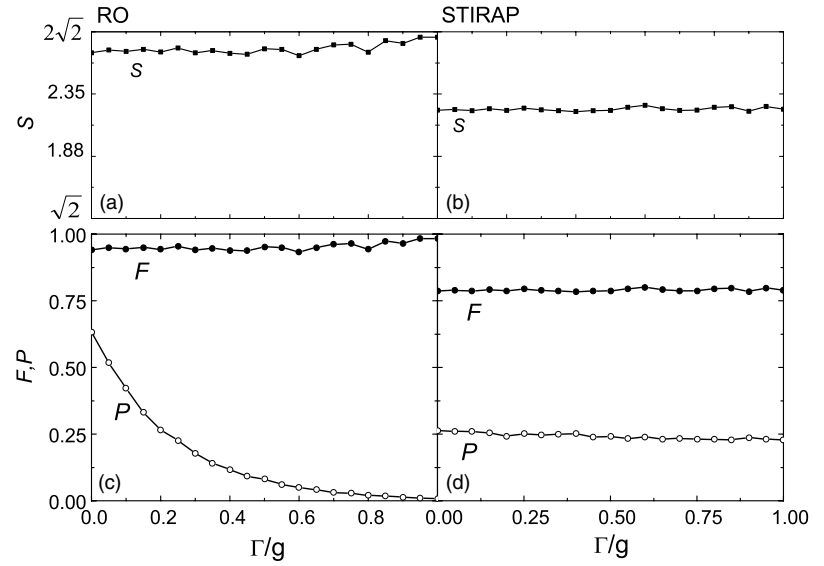

FIG. 4. The CHSH parameter $S$ [(a) and (b), see text $]$ and the success probability $P$ and fidelity $F[(\mathrm{c})$ and (d) $]$ as a function of the atomic decay for RO $(\mathrm{a}, \mathrm{c})$ and the STIRAP process $(\mathrm{b}, \mathrm{d})$. The parameters are $\Delta=0$ and $\kappa=0.053 g$.

due to the adiabaticity requirement. For state-of-the-art parameters, the RO and the STIRAP proposal have success probabilities of producing a pair of entangled photons after sending one atom through the cavities of around $40 \%$ and $20 \%$, respectively. However, most of the events not leading to an entangled pair of photons can be identified, and for such a post-selection process we have quantified the entanglement capability through the $S$ parameter of the $\mathrm{CHSH}$ inequality, which for the same parameters as before is $S=2.7$ (RO) and $S=2.3$ (STIRAP). This in both cases clearly exceeds the value $S=\sqrt{2}$ for separable states and the limit $S=2$ predicted by local hidden variable theories [28]. Finally, we illustrated the robustness of the STIRAP scheme under
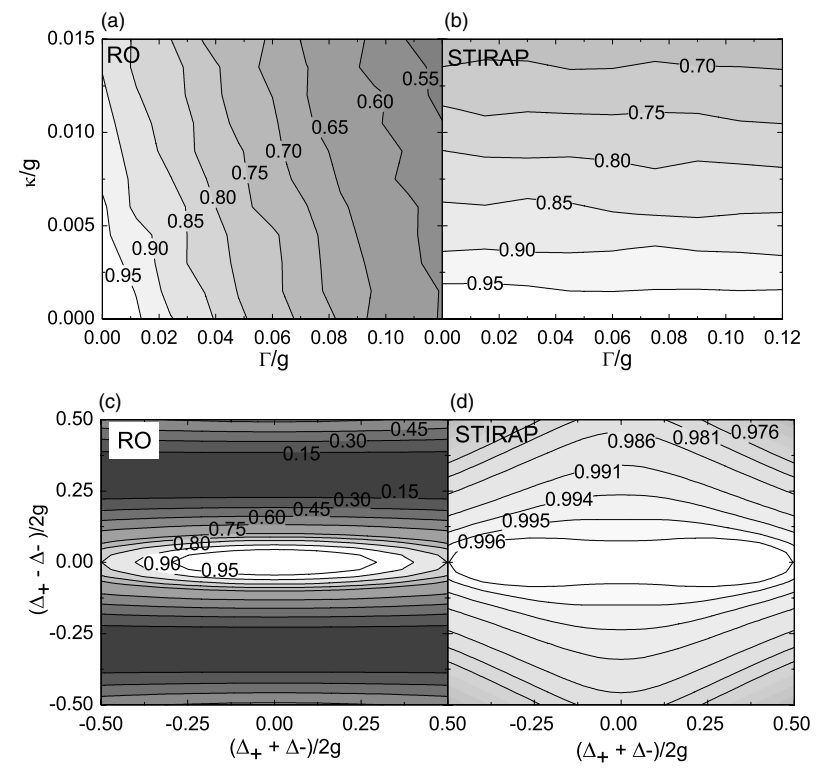

FIG. 5. Contour plots of the success probabilities for the RO scheme $(\mathrm{a}, \mathrm{c})$ and via STIRAP $(\mathrm{b}, \mathrm{d})$. Parameters are $\Delta_{+}=\Delta_{-}=0$ for $(\mathrm{a}, \mathrm{b})$ and $\kappa=\Gamma=0$ for $(\mathrm{c}, \mathrm{d}) .\left(\Delta_{+}+\Delta_{-}\right) / 2 g$ and $\left(\Delta_{+}-\Delta_{-}\right) / 2 g$ measure the deviation from the single- and the two-photon resonance condition, respectively.

fluctuations of experimental parameters as external magnetic fields.

We acknowledge support from Contracts No. FIS200501497MCyT (Spanish Government), No. SGR2005-00358 (Catalan Government), and No. EME2004-53 (Universitat Autònoma de Barcelona). K.E. acknowledges support received from the European Science Foundation PESC QUDEDIS.
[1] C. H. Bennet and G. Brassard, Proceedings of the IEEE International Conference on Computers, Systems, and Signal Processing (IEEE, New York, 1984), p. 175.

[2] V. Scarani et al., Phys. Rev. Lett. 92, 057901 (2004).

[3] A. K. Ekert, Phys. Rev. Lett. 67, 661 (1991).

[4] G. Brassard et al., Phys. Rev. Lett. 85, 1330 (2000).

[5] D. Mayers and A. Yao, Proceedings of the 39th IEEE Conference on Foundations of Computer Science (1998).

[6] G. Ribordy et al., Phys. Rev. A 63, 012309 (2000).

[7] T. Jennewein et al., Phys. Rev. Lett. 84, 4729 (2000).

[8] D. S. Naik et al., Phys. Rev. Lett. 84, 4733 (2000).

[9] W. Tittel et al., Phys. Rev. Lett. 84, 4737 (2000).

[10] D. L. Zhou et al., e-print quant-ph/0509165.

[11] L. Ye, L.-B. Yu, and G.-C. Guo, Phys. Rev. A 72, 034304 (2005).

[12] O. Benson et al., Phys. Rev. Lett. 84, 2513 (2000); C. Simon, J. P. Poizat et al., ibid. 94, 030502 (2005); N. Akopian et al., ibid. 96, 130501 (2006); R. M. Stevenson et al., Nature (London) 439, 179 (2006).

[13] G. Morigi et al., Phys. Rev. Lett. 96, 023601 (2006).

[14] C. H. Bennett et al., Phys. Rev. Lett. 70, 1895 (1993).

[15] C. H. Bennett et al., Phys. Rev. Lett. 69, 2881 (1992).

[16] A. Rauschenbeutel et al., Science 288, 2024 (2000).
[17] H. Walther, Fortschr. Phys. 51, 521 (2003).

[18] H. J. Kimble et al., Phys. Rev. Lett. 90, 249801 (2003).

[19] J. Gea-Banacloche et al., Phys. Rev. Lett. 94, 053603 (2005).

[20] A. Kuhn, M. Hennrich, and G. Rempe, Phys. Rev. Lett. 89, 067901 (2002).

[21] T. Legero et al., Phys. Rev. Lett. 93, 070503 (2004).

[22] T. E. Northup et al., in Atomic Physics 19, edited by L. G. Marcassa, V. S. Bagnato, and K. Helmerson (AIP, New York, 2004), Vol. 770, p. 313.

[23] J. A. Sauer et al., Phys. Rev. A 69, 051804(R) (2004).

[24] K. Bergmann, H. Theuer, and B. Shore, Rev. Mod. Phys. 70, 1003 (1998).

[25] For the setup of Fig. 1(b), the time dependence of the couplings $g_{i}(t)$ is determined by the transverse profile of the cavity modes and the atomic velocity.

[26] E. Arimondo, Prog. Opt. 35, 257 (1996).

[27] J. Dalibard, Y. Castin, and K. Mølmer, Phys. Rev. Lett. 68, 580 (1992).

[28] J. F. Clauser et al., Phys. Rev. Lett. 23, 880 (1969).

[29] For the processes including post-selection, the concurrence [30] takes values of $C=0.83$ (RO) and $C=0.53$ (STIRAP) for the parameters of Fig. 4 and $\Gamma=0.08 g$.

[30] S. Hill and W. K. Wootters, Phys. Rev. Lett. 78, 5022 (1997). 